\title{
Characterization of non albicans candida species and the antifungal susceptibility profile of clinical isolates
}

\author{
Lekshmi Balaraman', Saritha $\mathbf{N}^{2, *}$, Geetha Raveendran ${ }^{3}$ \\ ${ }^{1}$ Senior Resident, ${ }^{2}$ Associate Professor, Dept. of Microbiology, Government Medical College, KUHS, Thiruvananthapuram, \\ Kerala, ${ }^{3}$ Professor and Head, Dept. of Microbiology, Government Medical College, KUHS, Kollam, Kerala, India
}

*Corresponding Author:

Email: drsarithapradeep@gmail.com

Received: $28^{\text {th }}$ April, 2018

Accepted: $4^{\text {th }}$ July, 2018

\begin{abstract}
Introduction and Objectives: Antibiotics, immunodeficiencies and advances in medical practices have all contributed to increased incidence in fungal infections. In recent times Non albicans Candida species are increasingly being isolated from clinical specimens. The present study was aimed at the characterization and antifungal susceptibility of Non albicans Candida species from clinical specimens received in Microbiology Laboratory for one year from June 2015-May 2016.

Materials and Methods: A total of 181 Non albicans Candida isolates were included in the study. Candida was identified from clinical samples by gram stain, culture on Saboraud's Dextrose Agar (SDA) and germ tube test. The organisms were further speciated by cornmeal agar morphology, sugar assimilation and fermentation tests and growth on CHROM agar (Himedia). Antifungal sensitivity was performed by using disc diffusion method as per CLSI guidelines.

Results: The most common isolate was Candida tropicalis (59.11\%) followed by C.glabrata (28.18\%), C.parapsilosis (8.83\%) and C.krusei (3.88\%). Highest number of isolates were from urine (74/181). Resistance to Fluconazole in clinical isolates of C.tropicalis has increased. C.krusei was the most resistant species.

Conclusion: Use of CHROM agar is rapid, simple and cost effective compared to time consuming technically demanding expensive conventional methods. Antifungal susceptibility testing has to be carried out before starting therapy because of varying susceptibility pattern in different species of Candida.
\end{abstract}

Keywords: Non albicans Candida species, Candidiasis, CHROM agar.

\section{Introduction}

The incidence of candidiasis has increased dramatically over the past three decades. Increased therapeutic applications of immunosuppressive drugs, the use of broad spectrum antibiotics and the emergence of HIV/AIDS are important predisposing factors. Candida is capable of causing infections in both immunocompetent as well as immunocompromised hosts, but the incidence of candidiasis is more in immunocompromised individuals.

Therefore candidiasis can be rightly called as "disease of diseased". ${ }^{1}$ The incidence of Non albicans Candida is increasing compared with that of Candida albicans, and several species, such as Candida glabrata and Candida krusei, may be resistant to Azole antifungal therapy. ${ }^{2}$ The importance of early and accurate identification of infecting Candida species along with susceptibility testing for timely institution of appropriate therapy cannot be overstated. ${ }^{3}$

\section{Materials and Methods}

The isolates were identified by the following testsCulture (Inoculation on to Sabouraud's Dextrose Agar (Himedia), Cornmeal agar (Himedia), Hichrome Candida agar, Sugar assimilation test and Sugar Fermentation test. ${ }^{4,5}$

Culture: The isolates were subcultured onto two SDA tubes. The tubes were incubated at room temperature and at $37^{\circ} \mathrm{C}$. Candida colonies appear within 24 to 72 hours.

Dalmau Plate Culture (Inoculation into Cornmeal agar): Load a loop with the test organism and form a well at the top of a plate of cornmeal agar. Make dilution strokes by cutting into the agar with the edge of the sterilized loop. Dip a glass coverslip in alcohol, sterilize it by flaming, and let it cool; then put it on the agar to cover part of the well and some of the dilution strokes. Incubate at $22^{\circ} \mathrm{C}$ for 48 hours and examine by placing the plate on a microscope from which the slide carrier has been removed. Begin by scanning the area under the cover-slip and then, if no filamentous structures are found, carefully focus on the submerged growth that tracts along the incisions in the agar.

CHROMagar Candida: CHROMagar Candida is widely used for primary isolation and differentiation for clinical specimens likely to contain yeasts. This is based on the direct detection of specific enzymatic activities by adding certain substrates of fluorochromes to the media.

Carbohydrate Assimilation Test: The method consists essentially of growing a yeast on a basal carbohydrate-free medium supplemented with the test sugar. Yeast Nitrogen Base (YNB) was obtained from HiMedia. All the ingredients were mixed and autoclaved at $121^{\circ} \mathrm{C}$ for 15 minutes. Carbohydrate assimilation tests were performed by Auxanographic plate method. Yeast suspension was prepared by 
emulsifying colonies from a 24 hour old culture in $6 \mathrm{ml}$ of distilled water. The turbidity adjusted to McFarland No: 4. Two sterile glass petri dishes labelled with the isolate number and the names of the sugars were used. The Yeast suspension was poured equally into both the glass petridishes. $15 \mathrm{ml}$ of molten yeast nitrogen base cooled to $50^{\circ} \mathrm{C}$ was added to the yeast suspension and mixed well and allowed to set at room temperature. Carbohydrate disks of $4 \%$ concentration (Glucose, sucrose, lactose, maltose, galactose and trehalose) were evenly spaced in the designated area on the plates. The plates were incubated at $25^{\circ} \mathrm{c}$ for $24-48$ hours. The plates were observed for growth around the disks. Those isolates which assimilated a particular carbohydrate grow well around the disk. A negative assimilation test was reported when there was no opacity around the carbohydrate discs.

Sugar Fermentation Test: A loopful of culture was suspended in sterile distilled water. $0.2 \mathrm{ml}$ of this suspension was then added to $2 \%$ sugar fermentation media. Glucose, sucrose, lactose, maltose, galactose and trehalose were the sugars tested. The tubes were incubated at $30^{\circ} \mathrm{C}$ for 48 to 72 hours. The ability to ferment a sugar was shown by the presence of acid (colour change) and gas trapped in Durhams tube.

Antifungal Susceptibility Testing: Antifungal susceptibility test was done according to CLSI M44-A guidelines. Media used was Mueller Hinton Agar with $2 \%$ glucose and $0.005 \%$ methylene blue. The following antifungal discs were tested: Fluconazole $(25 \mu \mathrm{g})$, Itraconazole $(30 \mu \mathrm{g})$ Amphotericin B $(100 \mu \mathrm{g})$, Ketoconazole $(30 \mu \mathrm{g})$, Voriconazole $(1 \mu \mathrm{g})$. The control strains used were Candida parapsilosis ATCC 22019 and Candida tropicalis ATCC 750.

\section{Results}

About 626 samples were processed during the year. The number of Non albicans candida species were 181 and Candida albicans were 2 in number. Highest number of isolates were from urine. (Table 1) C.tropicalis was the predominant isolate, followed by C.glabrata. (Table 2)

Out of the 181 isolates studied, 139 were high risk group individuals.

Diabetes mellitus was the most common risk factor alone and as a coexistent condition along with sepsis (11 patients) and with intravascular catheters (6 patients). There were no patients with no obvious risk factors (Table 3). The rate of isolation of the Non albicans Candida species was more in females than in males (Table 4). The maximum number of patients were above 60 years. (Table 5).

Out of the 74 urine samples, C.tropicalis was the predominant species followed by C.glabrata, C.parapsilosis and C.krusei. Among the 52 HVS samples, C.glabrata was the predominant species followed by C.paraplisosis and C.krusei. C.tropicalis was the predominant species isolated from blood followed by C.glabrata. Among the 25 exudates, C.tropicalis was the predominant isolate. (Table 6).

Table 1: Distribution of isolates in various specimens

\begin{tabular}{|l|c|c|}
\hline \multicolumn{1}{|c|}{ Specimen } & Number & Percentage \\
\hline Urine & 74 & 40.89 \\
\hline $\begin{array}{l}\text { High vaginal swab } \\
\text { (HVS) }\end{array}$ & 52 & 28.73 \\
\hline Blood & 26 & 14.36 \\
\hline Exudate & 25 & 13.81 \\
\hline $\begin{array}{l}\text { Miscellaneous } \\
\text { (catheter tip) }\end{array}$ & 4 & 2.21 \\
\hline Total & $\mathbf{1 8 1}$ & $\mathbf{1 0 0}$ \\
\hline
\end{tabular}

Table 2: Distribution of Non albicans Candida species

\begin{tabular}{|l|c|c|}
\hline Species & Number & Percentage \\
\hline C.tropicalis & 107 & 59.11 \\
\hline C.glabrata & 51 & 28.18 \\
\hline C.parapsilosis & 16 & 8.83 \\
\hline C.krusei & 7 & 3.88 \\
\hline
\end{tabular}

Table 3: Predisposing factors in various patients

\begin{tabular}{|l|c|}
\hline \multicolumn{1}{|c|}{ Predisposing factors } & No. of patients \\
\hline Diabetes mellitus & 54 \\
\hline Chronic drug therapy & 23 \\
\hline Sepsis & 20 \\
\hline Immunosuppression & 19 \\
\hline Intravascular catheters & 11 \\
\hline Preterm/low birth weight & 8 \\
\hline $\begin{array}{l}\text { Intrauterine contraceptive } \\
\text { devices }\end{array}$ & 4 \\
\hline
\end{tabular}

Table 4: Distribution of isolates in relation with gender

\begin{tabular}{|l|c|c|}
\hline Gender & Number & Percentage \\
\hline Female & 95 & 52.49 \\
\hline Male & 86 & 47.51 \\
\hline
\end{tabular}

Table 5: Distribution of isolates according to age

\begin{tabular}{|l|c|}
\hline \multicolumn{1}{|c|}{ Age in years } & Number \\
\hline$\leq 10$ year & 14 \\
\hline $11-20$ & 8 \\
\hline $21-30$ & 19 \\
\hline $31-40$ & 27 \\
\hline $41-50$ & 28 \\
\hline $51-60$ & 30 \\
\hline$>60$ & 55 \\
\hline Total & 181 \\
\hline
\end{tabular}


Table 6: Distribution of isolates in various samples

\begin{tabular}{|c|c|c|c|c|}
\hline Specimen & \multicolumn{4}{|c|}{ Isolate } \\
\hline & C.tropicalis & C.glabrata & C.parapsilosis & C.krusei \\
\hline Urine & 53 & 9 & 8 & 4 \\
\hline HVS & 17 & 30 & 5 & 0 \\
\hline Blood & 19 & 7 & 0 & 0 \\
\hline Exudate & 15 & 4 & 3 & 3 \\
\hline Miscellaneous & 3 & 1 & 0 & 0 \\
\hline Total & 107 & 51 & 16 & 7 \\
\hline
\end{tabular}

Table 7: Distribution of isolates according to anti-fungal susceptibility pattern

\begin{tabular}{|l|c|c|c|c|c|c|c|c|c|c|}
\hline \multicolumn{1}{|c|}{ Isolates } & \multicolumn{2}{|c|}{ Fluconazole, $\mathbf{n}(\boldsymbol{\%})$} & \multicolumn{2}{|c|}{ Itraconazole, $\mathbf{n}(\boldsymbol{\%})$} & \multicolumn{2}{|c|}{$\begin{array}{c}\text { Amphotericin B, } \mathbf{n} \\
(\boldsymbol{\%})\end{array}$} & $\begin{array}{c}\text { Ketoconazole, } \mathbf{n} \\
(\boldsymbol{\%})\end{array}$ & \multicolumn{2}{|c|}{$\begin{array}{c}\text { Voriconazole, } \mathbf{n} \\
(\boldsymbol{\%})\end{array}$} \\
\hline & Sensitive & Resistant & Sensitive & Resistant & Sensitive & Resistant & Sensitive & Resistant & Sensitive & Resistant \\
\hline C.tropicalis & $81(75.70)$ & $26(24.3)$ & $88(82.24)$ & $19(18.67)$ & $102(95.33)$ & $5(4.67)$ & $95(88.79)$ & $12(11.21)$ & $104(97.2)$ & $3(2.8)$ \\
\hline C.glabrata & $6(11.76)$ & $45(88.24)$ & $13(25.49)$ & $38(74.51)$ & $40(78.43)$ & $11(21.57)$ & $23(45.1)$ & $28(54.9)$ & $36(70.59)$ & $15(29.41)$ \\
\hline C.parapsilosis & $14(87.5)$ & $2(12.5)$ & $15(93.75)$ & $1(6.25)$ & $16(100)$ & 0 & $15(93.75)$ & $1(6.25)$ & $16(100)$ & 0 \\
\hline C.krusei & 0 & $7(100)$ & 0 & $7(100)$ & $5(71.42)$ & $2(28.58)$ & $3(42.85)$ & $4(57.15)$ & $4(57.14)$ & $3(42.86)$ \\
\hline
\end{tabular}

C.parapsilosis was the most sensitive among the four isolates. All the isolates were fully sensitive to Voriconazole and Amphotericin-B. Out of the 16 isolates, $87.5 \%$ of isolates were sensitive to Fluconazole. C.krusei was the most resistant among the four isolates. All the 7 isolates were resistant to Fluconazole and Itraconazole, whereas $71.42 \%$ of isolates were sensitive to Amphotericin - B. All the antifungal discs were commercially obtained from Himedia. Disk tests are inexpensive and easy to set up, and provide an ideal screening test. The disk diffusion method to test antifungals has been developed and validated only in the case of azoles and echinocandins for Candida spp. isolates. For polyenes, MIC is recommended, which is a limitation of the study. Although qualitative results provided by the disk diffusion method are useful in the clinical laboratory routine, quantitative MIC data is somewhat critical for the management of invasive infections. (Table 7)

\section{Discussion}

The reported incidence of fungal infections associated with Non albicans Candida species is increasing in recent times. This is related largely to a dramatic increase in the number of individuals with deficient cellular immunity, particularly those infected with HIV and individuals receiving immunosuppressive treatments, both in organ transplantation and in anticancer therapy. ${ }^{6}$

About 181 Non albicans candida isolates and only two Candida albicans isolates were obtained during the year. Among the 181 isolates, C.tropicalis was the predominant isolate $(59.11 \%)$, followed by C.glabrata (28.18\%), C.parapsilosis (8.83\%), C.krusei (3.88\%). In the study conducted by Deorukhar et $\mathrm{al}^{7}$ in Maharashtra, India a total of 523 Candida spp. were isolated from various clinical specimens, Non albicans Candida species had a higher prevalence compared to C.albicans. Among the NAC spp., C. tropicalis
(35.1\%) was the predominant isolate followed by $C$. glabrata $(28.1 \%)$, which is in agreement with our study.

In our study, the highest incidence of candidiasis was found to be in persons more than 60 years. These findings were in concordance with the study conducted by Holley et $\mathrm{al}^{8}$ in France among 137 patients admitted in ICU, where the median age of patients was 63 years. This might be due to the co-morbid conditions like diabetes mellitus, renal failure, neoplastic disease, use of chemotherapeutic agents etc, which is common in this age group.

Female patients contributed $52.49 \%$ and male patients $47.51 \%$. Similar female predominance was noted by Kandhari KC et al (52.49\%), ${ }^{9}$ Dharwad et al $(64 \%)^{10}$ in Mangalore, India, where 100 candida isolates were taken for study. This could be due to the higher number of samples which were collected from female patients.

Among the risk factors, Diabetes mellitus was the most frequent. Similar findings were observed by Liao et $a l,{ }^{11}$ where a total of 306 proven ICU cases were included in the study. Candidemia incidence is statistically correlated with hyperglycemia. Severe hyperglycemia is considered as important risk factor of serious consequences viz increased morbidity, worst clinical course, longer hospital stay, late complications and mortality. ${ }^{12,13}$

C.tropicalis was the most common NAC isolated. $24.3 \%$ of isolates showed resistance to Fluconazole. Resistance to Fluconazole in clinical isolates of $C$. tropicalis has increased. Yang et $\mathrm{al}^{14}$ in Taiwan described a higher incidence of Fluconazole resistance in Candida tropicalis, where a total of 632 isolates were included in the study. The least resistance were observed with Voriconazole and Amphotericin-B. C.parapsilosis exhibited least resistance to antifungals. Similar results were reported by Fluckiger et $\mathrm{al}^{15}$ in Switzerland among 1137 isolates, where C.parapsilosis 
isolates were quite susceptible to most systemic antifungal agents including polyenes (Amphotericin B) and azoles (Fluconazole, Ketoconazole, Itraconazole, Voriconazole, and Posaconazole).

A higher rate of resistance to azoles were noted among C.glabrata isolates. $88.24 \%$ of isolates were resistant to Fluconazole, $74.51 \%$ of isolates were resistant to Itraconazole and $54.9 \%$ were resistant to Ketoconazole. Similar results were observed by Zomorodian et $\mathrm{a}^{16}$ in Iran where 206 clinical isolates of candida were included in the study, highest rates of resistance to Ketoconazole (16.6\%) and Fluconazole (96.6\%) were seen in Candida glabrata.

C.krusei was the most resistant among the four isolates. All the 7 isolates were resistant to Fluconazole and Itraconazole, where as $57.14 \%$ of isolates were sensitive to Voriconazole and $71.42 \%$ of isolates were sensitive to Amphotericin - B. Similar pattern were observed by Pfaller et al, ${ }^{17}$ where a decreased susceptibility to Fluconazole was noted.

Out of the 26 blood samples, 21 of them were from patients admitted in ICU. Among the 26 patients, 4 patients succumbed to death after starting antifungal therapy and 3 among them were awaiting culture reports. All the other patients were given systemic antifungal therapy and had improved outcome. Among the 52 patients with vulvovaginal candidiasis, 40 were given topical antifungal therapy. Those with intrauterine contraceptive devices (IUCD) were benefitted with removal of IUCD. Among the eight preterm infants, two had multiple comorbid conditions and were not benefitted with antifungal therapy.

\section{Conclusion}

During the last few years, the spectrum of infections has undergone a drastic change; organisms with minimal or no pathogenic role have emerged as potent pathogens and the organisms once susceptible have become multidrug resistant. A significant epidemiological shift to higher isolation of Non albicans Candida species was noticed. According to our study, there was an increase in the incidence and antifungal resistance of Non albicans Candida species, specifically C. krusei, C. glabrata and C. tropicalis, and the unacceptably high morbidity and mortality associated with these species, make it essential to further augment our knowledge on the virulence and resistance mechanisms associated with these species.

\section{References}

1. Deorukhkar S, Saini S. Virulence Markers and Antifungal Susceptibility Profile of Candida glabrata: An Emerging Pathogen. Br Microbiol Res J. 2014;4(1):39-49.

2. Miceli MH, Diaz JA, Lee SA. Emerging opportunistic yeast infections. Lancet Infect Dis. 2011;11(2):142-51.

3. Jaggi T, Urhekar AD, Pai C, Hodiwala AB, Gore S, Kar H. Study of Candida Species in Various Clinical Samples in a Tertiary Care Hospital. DHR Int J Med Sci. 2014;5(2):2278-83.

4. Deorukhkar SC, Saini S. Laboratory approach for diagnosis of candidiasis through ages. Int J Curr Microbiol App Sci. 2014;3(1):206-218.

5. Collee JG, Fraser AG, Marmion BP, Simmons A. Mackie and McCartney Practical Medical Microbiology. Churchill Livingstone. London 2014. 14th edn:699-700

6. Sullivan DJ, Henmans MC, Moran GI, Lorraine C O'Neill, Bennett DE, Shanley DB et al. Molecular genetic approaches to identification, epidemiology and taxonomy of Non albicans Candida species. J Med Microbiol. 1996;44:399-408.

7. Deorukhkar SC, Saini S, and Mathew S. Non albicans Candida Infection: An Emerging Threat Article ID 615958. Interdiscip Perspect Infect Dis. 2014: 1-7.

8. Holley A, Dulhunty J, Blot S, Lipman J, Lobo S, Dancer $\mathrm{C}$ et al. Temporal trends, risk factors and outcomes in albicans and non-albicans candidaemia: an international epidemiological study in four multidisciplinary intensive care units. Int J Antimicrob Agents. 2009;554:1-7.

9. Khandari KC, Rama KM. Clinical and laboratory studies on cutaneous candidiasis. MD J Der Ven Lep. 1969;35(2):102-107.

10. Dharwad S, Dominic S. Species Identification of Candida Isolates in Various Clinical Specimens with their Antifungal Susceptibility Patterns. J Clin Diagn Res. 2011;5(6):1177-81.

11. Liao X, Qiu H, Li R, Guo F, Liu W, Kang M et al. Risk factors for Fluconazole-resistant invasive candidiasis in intensive care unit patients: An analysis from the China Survey of Candidiasis study. J Crit Care. 2015;30:1-5.

12. Bader MS, Hinthorn D, Lai SM. Diabet Med. 2005;22:1252-7.

13. Pfaller MA, Diekema DJ. J Clin Microbiol. 2004;4:441931.

14. Yang YL, Ho YA, Cheng HH., Ho M, Lo HJ. Susceptibilities of Candida species to Amphotericin B and Fluconazole: the emergence of Fluconazole resistance in Candida tropicalis. Infect Control Hosp Epidemiol. 2004;25:60-4.

15. Fluckiger U, Marchetti O, Bille J, Eggimann P, Zimmerli $\mathrm{S}$, Imhof A et al. Treatment options of invasive fungal infections in adults. Swiss Med Wkly. 2006;136:447-63.

16. Zomorodian K, Rahimi MJ, Pakshir K, Motamedi M, Ghiasi MR, Rezashah H. Determination of antifungal susceptibility patterns among the clinical isolates of Candida species. J Global Infect Dis. 2011;3:357-60.

17. Pfaller MA, Diekema DJ, Gibbs DL, Newell VA, Nagy E, Dobiasova S et al and the Global Antifungal Surveillance Group. Candida krusei, a MultidrugResistant Opportunistic Fungal Pathogen: Geographic and Temporal Trends from the ARTEMIS DISK Antifungal Surveillance Program, 2001 to 2005. J Clin Microbiol. 2008;46(2):515-21.

How to cite this article: Balaraman L, Saritha $\mathrm{N}$, Raveendran G. Characterization of non albicans candida species and the antifungal susceptibility profile of clinical isolates. Indian J Microbiol Res. 2018;5(3):295-298. 